\title{
BMJ Global Health Prevalence of chronic kidney disease in Asia: a systematic review and analysis
}

\author{
Thaminda Liyanage, ${ }^{1,2,3}$ Tadashi Toyama, ${ }^{4}$ Carinna Hockham, ${ }^{5}$ \\ Toshiharu Ninomiya, ${ }^{6}$ Vlado Perkovic, ${ }^{1,7}$ Mark Woodward, ${ }^{1,5,8}$ \\ Masafumi Fukagawa, ${ }^{9}$ Kunihiro Matsushita, ${ }^{8}$ Kearkiat Praditpornsilpa, ${ }^{10}$ \\ Lai Seong Hooi, ${ }^{11}$ Kunitoshi Iseki, ${ }^{12}$ Ming-Yen Lin (DD ,13 \\ Heide A. Stirnadel-Farrant, ${ }^{14}$ Vivekanand Jha, ${ }^{15,16,17}$ Min Jun ${ }^{1,7}$
}

To cite: Liyanage T, Toyama T, Hockham C, et al. Prevalence of chronic kidney disease in Asia: a systematic review and analysis. BMJ Global Health 2022;7:e007525. doi:10.1136/ bmjgh-2021-007525

Handling editor Sanne Peters

TL and TT contributed equally.

Received 27 September 2021 Accepted 25 December 2021

Check for updates

(c) Author(s) (or their employer(s)) 2022. Re-use permitted under CC BY-NC. No commercial re-use. See rights and permissions. Published by BMJ

For numbered affiliations see end of article.

Correspondence to

A/Prof Min Jun;

mjun@georgeinstitute.org.au

\section{ABSTRACT}

Introduction The burden of chronic kidney disease (CKD) is growing rapidly around the world. However, there is limited information on the overall regional prevalence of CKD, as well as the variations in national prevalence within Asia. We aimed to consolidate available data and quantify estimates of the CKD burden in this region.

Methods We systematically searched MEDLINE, Embase and Google Scholar for observational studies and contacted national experts to estimate CKD prevalence in countries of Asia (Eastern, Southern and South Eastern Asia). CKD was defined as estimated glomerular filtration rate (eGFR) $<60 \mathrm{~mL} / \mathrm{min} / 1.73 \mathrm{~m}^{2}$ or the presence of proteinuria. For countries without reported data, we estimated CKD prevalence using agglomerative averagelinkage hierarchical clustering, based on country-level risk factors and random effects meta-analysis within clusters. Published CKD prevalence data were obtained for 16 countries (of the 26 countries in the region) and estimates were made for 10 countries.

Results There was substantial variation in overall and advanced (eGFR $<30 \mathrm{~mL} / \mathrm{min} / 1.73 \mathrm{~m}^{2}$ ) CKD prevalence (range: $7.0 \%-34.3 \%$ and $0.1 \%-17.0 \%$, respectively). Up to an estimated 434.3 million $(95 \% \mathrm{Cl} 350.2$ to 519.7$)$ adults have CKD in Asia, including up to 65.6 million $(95 \% \mathrm{Cl} 42.2$ to 94.9) who have advanced CKD. The greatest number of adults living with CKD were in China (up to 159.8 million, $95 \% \mathrm{Cl} 146.6$ to 174.1 ) and India (up to 140.2 million, $95 \% \mathrm{Cl} 110.7$ to 169.7 ), collectively having $69.1 \%$ of the total number of adults with CKD in the region.

Conclusion The large number of people with CKD, and the substantial number with advanced CKD, show the need for urgent collaborative action in Asia to prevent and manage CKD and its complications.

\section{INTRODUCTION}

Chronic kidney disease (CKD), typically defined as glomerular filtration rate (GFR) $<60 \mathrm{~mL}$ / $\min / 1.73 \mathrm{~m}^{2}$ or the presence of other markers of kidney deterioration such as albuminuria, ${ }^{1}$ is a major public health problem worldwide. ${ }^{2}$ CKD is also strongly interrelated with other major

\section{Key questions}

What is already known about this subject?

- Chronic kidney disease (CKD) is a major public health problem globally and with the rising prevalence of diabetes and hypertension (the leading causes of CKD) worldwide, CKD prevalence is projected to increase continually.

- Much of this increasing burden is expected to be observed in Asia, the most populous continent with a population of $>4.5$ billion people $(60 \%$ of the global population)

- Accumulating data suggest that there is substantial variation in CKD prevalence across the region, however, a comprehensive assessment of the prevalence of CKD and the estimation of the absolute number of people with CKD in Asia has been lacking.

What are the new findings?

- This study was conducted through the Asian Renal Collaboration, a group of international investigators representing and contributing cohort study data on people with CKD from the Asia-Pacific region. To our knowledge, this represents the first collaborative review to consolidate available data on CKD prevalence in Asia.

- Our results show substantial variation in CKD prevalence $(7.0 \%-34.3 \%)$ across Asia. We estimate that up to approximately 434.3 million people have CKD across the eastern, southern and south-eastern regions of Asia, with most of the disease burden observed in China and India (up to 299.9 million). Of the overall number of people with CKD, our findings suggest that up to 65.6 million have advanced stages of CKD.

\section{What do the new findings imply?}

- Overall, our results emphasise the substantial burden of CKD in Asia.

- These data highlight the increasing importance of early detection (including potentially screening for CKD) and treatment of CKD in Asia, particularly given that achieving meaningful improvements in clinical outcomes in individuals with already advanced CKD has shown to be challenging. 
diseases, including diabetes and cardiovascular disease, which remain the leading causes of morbidity and premature death in this patient population. ${ }^{3}$

CKD burden is projected to continually increase internationally, ${ }^{4}$ driven by the rising prevalence of diabetes, which is the leading cause of CKD globally. Much of this increasing burden is expected to occur in Asia, ${ }^{5}$ a region that is home to $>4.5$ billion people or $60 \%$ of the global population. In South Asia alone, diabetes prevalence is estimated to increase by over $150 \%$ between 2000 and $2035^{67}$; while China and India are projected to remain as the countries with the highest number of people with diabetes by 2035 (a total of 251.7 million people). ${ }^{6}$ In addition, increasing clusters of CKD of unknown aetiology have been reported in parts of South Asia including Sri Lanka ${ }^{8}$ and India. ${ }^{9}$ Combined with population ageing in Asia, ${ }^{10}$ these data indicate an increasing burden of CKD in Asia. However, data on contemporary CKD prevalence across this region to better inform future projections have been relatively sparse, while kidney registries in Asia have mostly restricted systematic data collection to patients with kidney failure requiring kidney replacement therapy. ${ }^{11}$ A number of individual reports-from the eastern, southern and south-eastern parts of Asia (eg, China, India and Vietnam) in particular-suggest a wide variation in the CKD prevalence across the region $(4.7 \%-17.4 \%) .{ }^{12-14} \mathrm{~A}$ comprehensive systematic overview of CKD prevalence specifically in Asia is needed to better delineate the burden of CKD and develop region-specific strategies aimed at the prevention and management of CKD in this region.

Through the Asian Renal Collaboration (ARC), a group of international investigators representing and contributing cohort study data on people with CKD from the Asia-Pacific region, ${ }^{15}$ we conducted a systematic review to (1) consolidate available data on CKD prevalence in Asia (defined as Eastern Asia, Southern Asia and SouthEastern Asia $^{16}$ and (2) further estimate CKD prevalence in countries of Asia where no reported data exist.

\section{METHODS}

\section{Search strategy and selection criteria}

We performed a systematic review of the literature describing CKD prevalence in countries of Asia (defined as Eastern Asia, Southern Asia and South-Eastern Asia), as per methods described in the prespecified study protocol for the ARC study ${ }^{15}$ and according to the Meta-analysis of Observational Studies in Epidemiology (MOOSE) group consensus statement. ${ }^{17}$ CKD was defined as estimated glomerular filtration rate $(\mathrm{eGFR})<60 \mathrm{~mL} / \mathrm{min} / 1.73 \mathrm{~m}^{2}$ or the presence of proteinuria according to the Kidney Disease Improving Global Outcomes (KDIGO) Clinical Practice Guideline. ${ }^{18}$ Advanced CKD was defined as eGFR $<30 \mathrm{~mL} / \mathrm{min} / 1.73 \mathrm{~m}^{2}$ (KDIGO G stages 4 and 5 ). In brief, cross-sectional or longitudinal cohort studies reporting prevalence and/or clinical data on people with CKD in a population from the Asia-Pacific region were eligible for inclusion. Eligible studies were identified through (1) a systematic literature search of MEDLINE via Ovid (1946 to January 2020; without language restriction), Embase (1947 to January 2020; without language restriction) and Google Scholar (to October 2020) searches based on relevant text words and medical subject headings related to Asian countries, kidney function and proteinuria (online supplemental appendix 1 and 2) contacting experts in the region ${ }^{15}$ for access to relevant individual or summary level participant data. Included studies involved $\geq 500$ adult (age $\geq 18$ years) participants. Reference lists from identified articles were also manually searched to identify any other relevant studies.

\section{Data extraction and quality assessment}

Titles, abstracts and full text articles (if necessary) were assessed independently by two authors (TL and TT) to determine which studies met the inclusion criteria for the systematic review. Data extraction was conducted independently by three authors (TL, TT and $\mathrm{CH}$ ) using a standard data extraction form. Disagreements in abstract screening and extracted data were adjudicated by another reviewer (VP or MJ). For studies which agreed to provide additional and/or updated summary or individual participant level data, information was collected using a standardised central data request form. ${ }^{15}$ The data sought included sociodemographic (age at study inclusion, gender, ethnicity, occupation and education), comorbid and laboratory (history of stroke and coronary heart disease, systolic and diastolic blood pressure, diabetes, lipid profile, obesity, serum creatinine, eGFR (calculated using the MDRD ${ }^{19}$ or CKD-EPI creatinine equation ${ }^{20}$ or as defined by authors), haemoglobin, iron status, calcium, phosphate, parathyroid hormone and vitamin D levels), medication use (eg, antihypertensive and erythropoietin stimulating agents) and CKD prevalence.

In addition to study and participant-related data, we also obtained country-specific information for 26 Asian countries (table 1), including total population estimates from the United Nations Population Division ${ }^{16}$ (number of people aged $\geq 20$ years in 2020) and national estimates of life expectancy at birth, gross national income (GNI) and prevalence of key risk factors of CKD (prevalence of diabetes, hypertension (blood pressure $\geq 140 / 90 \mathrm{~mm}$ $\mathrm{Hg}$ ) and obesity (body mass index $\geq 30 \mathrm{~kg} / \mathrm{m}^{2}$ )) from the WHO Global Health Observatory data repository. ${ }^{21} \mathrm{Popu}-$ lation data on Taiwan were obtained from the National Statistics (Taiwan) website. ${ }^{22} 23$

Study quality was judged independently by two authors (TL and TT) based on a modified version of the Newcastle-Ottawa Quality Assessment Scale for cohort studies. ${ }^{24}$ The criteria assessed were: (1) representativeness of the sample, (2) sample size ( $<3000$ vs $\geq 3000$ participants; based on the median study size of the included studies), (3) comparability of cohort (CKD vs no CKD) characteristics, (4) outcome ascertainment and (5) reporting of descriptive statistics. For each domain on 
Table 1 Country-level sociodemographic characteristics and risk factor prevalence of countries in Asia

\begin{tabular}{|c|c|c|c|c|c|}
\hline Countries & $\begin{array}{l}\text { Life expectancy at } \\
\text { birth* (years) }^{*}\end{array}$ & $\begin{array}{l}\text { GNI per capita† } \\
\text { (US\$) }\end{array}$ & $\begin{array}{l}\text { Prevalence of } \\
\text { diabetes } ¥(\%)\end{array}$ & $\begin{array}{l}\text { Prevalence of } \\
\text { BP§ } \geq 140 / 90 \mathrm{~mm} \mathrm{Hg} \mathrm{( \% )}\end{array}$ & $\begin{array}{l}\text { Prevalence of } \\
\text { BMI } \geq 30 \mathrm{~kg} / \mathrm{m}^{2}(\%)\end{array}$ \\
\hline Afghanistan & 63.2 & 520 & 11.9 & 30.6 & 5.5 \\
\hline Bangladesh & 74.3 & 1750 & 9.8 & 24.7 & 3.6 \\
\hline Bhutan & 73.1 & 2970 & 11.8 & 28.1 & 6.4 \\
\hline Brunei & 74.3 & 29390 & 9.4 & 18.9 & 14.1 \\
\hline Cambodia & 70.1 & 1380 & 7.1 & 26.1 & 3.9 \\
\hline China & 77.4 & 9600 & 8.8 & 19.2 & 6.2 \\
\hline India & 70.8 & 2010 & 8.7 & 25.8 & 3.9 \\
\hline Indonesia & 71.3 & 3850 & 7.7 & 23.8 & 6.9 \\
\hline Iran & 77.3 & 5300 & 12.1 & 19.7 & 25.8 \\
\hline Japan & 84.3 & 41150 & 6.7 & 17.6 & 4.3 \\
\hline Laos & 68.5 & 2450 & 7.7 & 24.8 & 5.3 \\
\hline Malaysia & 74.7 & 10650 & 11.1 & 22.9 & 15.6 \\
\hline Maldives & 79.6 & 9210 & 10.9 & 24.4 & 8.6 \\
\hline Mongolia & 68.1 & 3630 & 11.7 & 29 & 20.6 \\
\hline Myanmar & 69.1 & 1370 & 7.4 & 24.6 & 5.8 \\
\hline Nepal & 70.9 & 970 & 10.6 & 29.4 & 4.1 \\
\hline North Korea & 72.6 & No data & 5.9 & 18.2 & 6.8 \\
\hline Pakistan & 65.6 & 1480 & 12.4 & 30.5 & 8.6 \\
\hline Philippines & 70.4 & 3710 & 7.2 & 22.6 & 6.4 \\
\hline Singapore & 83.2 & 57900 & 7.9 & 14.6 & 6.1 \\
\hline South Korea & 83.3 & 32730 & 8 & 11 & 4.7 \\
\hline Sri Lanka & 76.9 & 4040 & 7.4 & 22.4 & 5.2 \\
\hline Taiwan & $80.9^{\star *}$ & $26,594^{\star \star}$ & 6.6†† & $24.1 \neq \ddagger$ & $8.2 \S \S$ \\
\hline Thailand & 77.7 & 6600 & 8.5 & 22.3 & 10 \\
\hline Timor-Leste & 69.6 & 1800 & 5.5 & 27.6 & 3.8 \\
\hline Viet Nam & 73.7 & 2385 & 5.3 & 23.4 & 2.1 \\
\hline
\end{tabular}

*Based on data from the 2019 WHO World Health Data Platform.

†Based on data from The World Bank (2019 GNI per capita).

‡Diabetes was defined as fasting glucose $\geq 7.0 \mathrm{mmol} / \mathrm{L}(126 \mathrm{mg} / \mathrm{dL})$, using glucose-lowering agents, and/or the presence of a history of diagnosis of diabetes (based on data from the 2014 WHO World Health Data Platform).

§Based on data from the 2015 WHO World Health Data Platform.

IB Based on data from the 2016 WHO World Health Data Platform.

${ }^{* *}$ National Statistics Republic of China (Taiwan).

††International Diabetes Federation.

抽aiwan Ministry of Health and Welfare.

$\S \S$ World Obesity Federation.

$\mathrm{BMI}$, body mass index; BP, blood pressure; GNI, gross national income.

the scale, the study received a score of 0 (indicating the study did not meet the criteria requirement or there was insufficient information to determine whether criteria requirement was met) or 1 (indicating the study met the criteria requirement). Low risk was defined as $4-5$ points, moderate risk as 3 points and high risk of bias was defined as $0-2$ points.

\section{Data synthesis and analysis}

For countries in which overall CKD prevalence data were available, the total number of people with CKD was estimated by multiplying the prevalence of CKD by the total adult population in 2020 in each of these countries. For countries in which more than one study was available, random-effects meta-analysis was used to estimate the pooled country-specific CKD prevalence. Where available, this was repeated to separately estimate the number of adults with advanced CKD in the included countries.

To allow for estimation of CKD prevalence (and the corresponding absolute number of people with CKD) in countries for which specific data were lacking, we conducted agglomerative average-linkage hierarchical cluster analysis, a statistical method that aims to assess 
for unknown characteristics of defined groups based on clustering according to known clinical and/or other attributes (eg, diabetes and GNI) ${ }^{25-28}$ This method is based on a 'bottom-up' approach where each observation (ie, country) is initially assigned to a separate cluster. Clusters with similar attributes (ie, the average 'distance' between clusters is short) are then sequentially combined until only a single cluster remains. In this analysis, we assumed that the magnitude of CKD prevalence is proportional to the presence of conventional CKD risk factors (diabetes, hypertension and obesity) and other country-level variables (national estimates of life expectancy and GNI per capita) such that countries with similar health and country-level attributes will have similar CKD prevalence.

Specifically, in estimating CKD prevalence, we (1) standardised the prevalence of clinical risk factors (diabetes, hypertension and obesity) and country-specific characteristics (life expectancy at birth and GNI per capita) to z scores (ie, number of SD a value is from predicted mean) to allow comparison across countries, (2) grouped the 26 countries into clusters (online supplemental appendix 2) based on similarity in country-level characteristics and (3) conducted random-effects meta-analysis within each cluster containing $\geq 3$ countries to estimate the pooled CKD prevalence in each cluster.

The cluster-specific summary CKD prevalence estimate (and the corresponding 95\% CIs) was used to derive the number of people with CKD in countries (in the relevant cluster) for which specific data were lacking. For clusters which contained two countries, only one of which lacked data, the number of people with CKD in the country for which CKD prevalence data were unavailable was estimated based on the known CKD prevalence of the other country. In countries in which overall CKD prevalence data were unavailable, the prevalence of advanced CKD reported in the eligible studies was used instead. The total number of people (and the corresponding 95\% CIs) with CKD in Asia were approximated as the sum of the estimated number of people with CKD for each of the individual countries assessed.

To account for differences in study inclusion criteria across the eligible studies (and its potential impact on reported CKD prevalence), for each country, we developed two sets of estimates by grouping eligible studies defined as 'population-based' and 'all studies'. We defined 'population-based' studies as eligible community-based studies which included a national or regional population of adults ( $\geq 18$ years) without specifying the presence of specific diseases or conditions (eg, diabetes or hypertension) in their inclusion criteria. 'All studies' included all eligible studies identified for each country in the current review.

To test for robustness of our study findings, we performed sensitivity analysis restricting study inclusion to more recently published studies (published between 2010 and 2020). All analyses were performed using Stata (V.14.1; StataCorp).

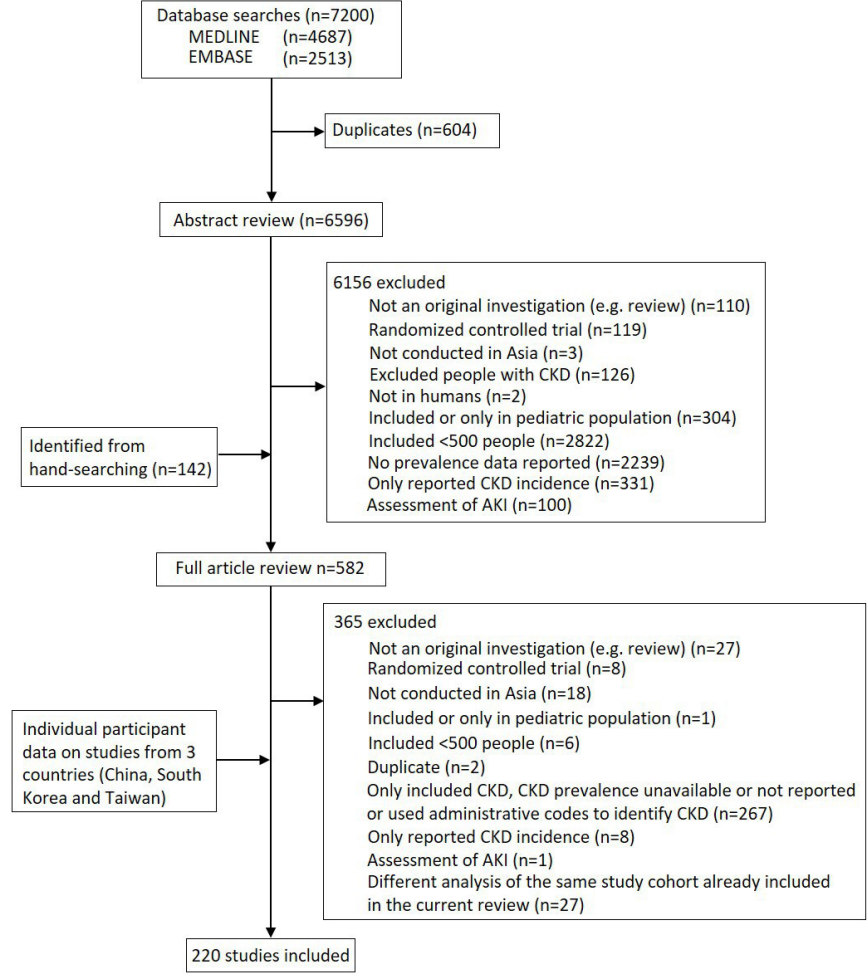

Figure 1 Identification of the included studies. CKD, chronic kidney disease. AKI, acute kidney injury

\section{Patient involvement}

This study involved the secondary use of existing or published data and did not include patients as study participants. No patients were involved in setting the research question, the study design or the overall conduct of the study. There are no plans to involve patients in the dissemination of study findings.

\section{RESULTS}

\section{Search results and characteristics of included studies}

Our search strategy identified 7200 articles from which 582 were selected for full text review (figure 1). Of these, 220 studies met the inclusion criteria (figure 1), including a total of 7233151 participants. This includes additionally obtained data on cohorts from China (summary level data; $\mathrm{n}=46968,{ }^{12}$ South Korea (individual participant data; $\mathrm{n}=5428^{29}$ and Taiwan (individual participant data; $\mathrm{n}=3142 .{ }^{30}$ Study size ranged from 504 to 2969502 participants (online supplemental appendix 3). Of the 220 included studies, 155 were cross-sectional studies and 65 were prospective cohort studies (median follow-up period of 2.7 years). Eighty-seven studies $(40.5 \%)$ defined CKD based on a combination of both eGFR and proteinuria (or albuminuria) measurements, while 128 (59.5\%) studies defined CKD based on eGFR or proteinuria (or albuminuria) alone. Most studies used a single measurement of eGFR and/or proteinuria assessment to define prevalence (195 studies; 90.3\%) and CKD screening was performed in 33 studies $(15 \%)$. One hundred and twenty-one (55\%), $51(23.2 \%)$ and $48(21.8 \%)$ studies 
used the MDRD, CKD-EPI or other equations (including Cockroft-Gault), respectively, to estimate glomerular filtration rate. The mean age of the study participants ranged between 20.2 and 86.0 years while the proportion of women ranged between $0 \%$ and $77 \%$. A summary of the study quality assessment is provided in online supplemental appendix 4 . Ninety-nine $(45.8 \%)$ had a low risk of bias, $76(35.2 \%)$ had a moderate risk of bias and 41 $(18.9 \%)$ had a high risk of bias.

\section{Prevalence of CKD in Asia}

Overall, of the 26 countries in the region, we obtained CKD prevalence data on cohorts from 16 countries; table 2). We obtained CKD prevalence data on cohorts from five countries in Eastern Asia (98.6\% of the adult Eastern Asian population), six countries in Southern Asia $(98.6 \%)$ and five countries in South-Eastern Asia (72\%). There were noticeable differences in catchment areas assessed (national vs regional), formulae used to estimate GFR and in the definition of CKD. The completeness of data collection and the robustness in reporting varied considerably among included studies. Online supplemental appendix 2 shows the results of country clustering. Mongolia and Iran were considered individually as these two countries had substantially different risk factor profiles to the other assessed countries (online supplemental appendix 2).

Reported CKD prevalence varied widely across the 16 countries with data, ranging from $7.0 \%(95 \%$ CI $5.1 \%$ to $8.9 \%$ ) in South Korea to $34.3 \%$ (95\% CI $0.0 \%$ to $71 \%$ ) in Singapore among population-based studies (range across all studies: $7.8 \%$ (95\% CI $6.2 \%$ to $9.5 \%$ ) in Mongolia to $33.2 \%$ (95\% CI $22.4 \%$ to $44.1 \%$ ) in Singapore; table 2). Advanced CKD ranged from $0.1 \%(95 \%$ CI $0.1 \%$ to $0.2 \%$ ) in Viet Nam to $17.0 \%$ (95\% CI $15.2 \%$ to $18.8 \%$ ) in both Afghanistan and Pakistan among populationbased studies (range across all studies: $0.1 \%$ (95\% CI $0.1 \%$ to $0.2 \%$ in Viet Nam to $4.5 \%$ (95\% CI $0.0 \%$ to $11.8 \%$ ) in Bangladesh). The prevalence of proteinuria or albuminuria also varied widely, ranging from $0.5 \%$ in Taiwan to $83.5 \%$ in Sri Lanka (online supplemental appendix 3). We estimated the prevalence of CKD in 10 countries from where no data were available (total population size of people aged $\geq 20$ years: 153.8 million, $5.2 \%$ of the total population of Asia): one in Eastern Asia (North Korea), three in Southern Asia (Afghanistan, Bhutan and Maldives) and six in South-Eastern Asia (Brunei, Cambodia, Laos, Myanmar, Philippines and Timor-Leste).

In total, up to an estimated 434.3 million (95\% CI 350.2 to 519.7 million) adults had CKD in Eastern, Southern and South-Eastern Asia (315.2 million (95\% CI 236.0 to 396.1 million) based on population-based studies only) this included 65.6 million (95\% CI 42.2 to 94.9 million) with advanced CKD (45.8 million (95\% CI 27.2 to 67.6 million) based on population-based studies only). As expected, the absolute number of people with CKD was estimated to be highest in China (up to 159.8 million
(95\% CI 146.6 to 174.1$)$ ) and India (up to 140.2 million (95\% CI 110.7 to 169.7 million); table 2), as was the absolute number of people with advanced CKD (China: up to 26.4 million (95\% CI 22.0 to 31.9 million); India: up to 17.9 million (95\% CI 11.6 to 24.1 million)). The estimated number of people with CKD and advanced CKD in the remaining 24 countries was up to 134.4 million and 21.3 million, respectively. Overall results remained unchanged in sensitivity analysis limited to more recently published studies (between 2010 and 2020; online supplemental appendix 5).

\section{DISCUSSION}

In this systematic review, we used the best available data to estimate the overall prevalence and the absolute number of people with CKD in Asia. We observed substantial variation in CKD prevalence estimates that ranged between $7.0 \%$ and $34.3 \%$ and estimate that approximately up to 434 million people have CKD across the eastern, southern and south-eastern regions of Asia. Additionally, our findings suggest that up to an estimated 65 million have more advanced forms of CKD (stages 4-5). Overall, these data highlight the substantial burden of CKD in Asia that may be prevalent at similar or even greater magnitudes in some Asian regions (eg, China and India) as compared with those observed in other parts of the world including North America and Europe. Taken together, our results highlight the importance of early detection and treatment of CKD in Asia, particularly given that achieving meaningful improvements in clinical outcomes in individuals with already advanced CKD has shown to be challenging.

Recently, the Global Burden of Disease (GBD) study reported that in 2017, 697.5 million (95\% uncertainty interval: 649.2-752.0 million) cases of CKD were recorded globally, with an overall prevalence of $9.1 \% .^{31}$ Overall estimates for Asia in the GBD study were relatively comparable to those observed in our study ( $\sim 434$ million vs $\sim 387$ (95\% CI 365 to 532 million) in the current and GBD study, respectively). However, several differences in country-specific CKD prevalence estimates between the two studies are notable. For example, estimates for Indonesia were higher in the GBD study ( 15.4 million vs $\sim 27.2$ million, respectively), while lower for Taiwan ( $\sim 4.4$ million vs $\sim 2.7$ million, respectively). Differences in estimates between the two studies may be attributable to a number of factors such as differences in study inclusion criteria, the time point at which the literature search was performed, and analytical methodology used. Another recent qualitative systematic review assessing the prevalence of CKD specifically in South Asia reported similar levels of CKD burden across Bangladesh, India, Nepal and Pakistan. ${ }^{32}$ Collectively, these data underline the high burden of CKD in Asia. Of note, our review, based on an updated literature search, further indicates that a substantial number of people in this region have advanced stages of CKD and suggests that strategies to improve outcomes 


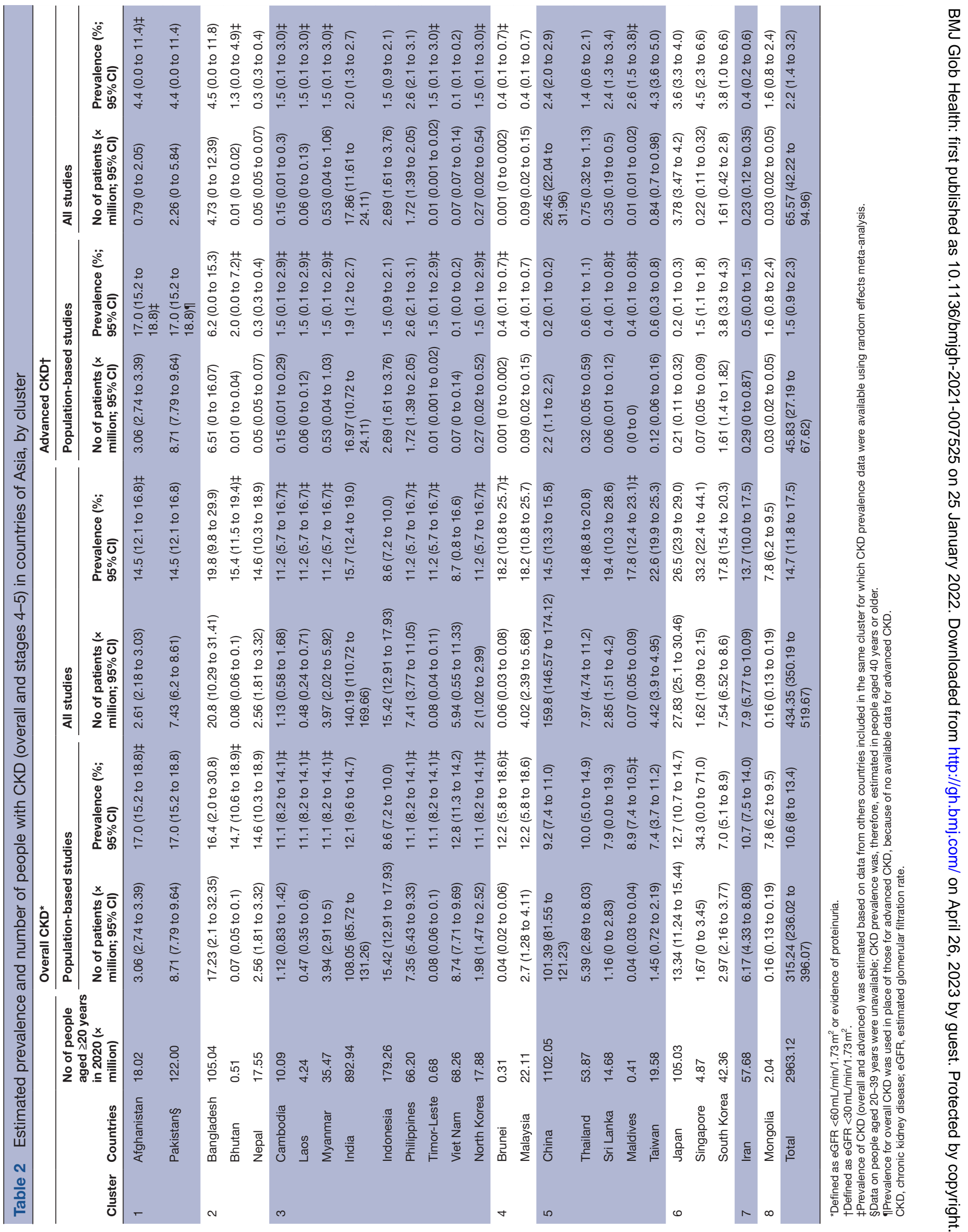


in this particularly high-risk group (eg, improved access to dialysis care) should be prioritised.

We observed substantial variation in CKD prevalence across Asia, which may be explained by several factors. First, there is substantial variability in the etiological spectrum of CKD in the region. Chronic glomerulonephritis (eg, IgA nephropathy) remains the leading cause of CKD in several countries in Asia while infectious disease (eg, hepatitis)-related CKD or those arising from unknown causes are highly prevalent in other parts of the region. In north and east Asia, it is postulated that relatively rapid changes in various sociodemographic and lifestyle factors known to drive major chronic disease prevalence has led to significant shifts in diabetic nephropathy prevalence in the region, ${ }^{33}$ comparable to estimates observed in other parts of the world where diabetic nephropathy typically predominates as the leading cause of CKD. Several clusters with high prevalence of CKD have been reported from different parts of South Asia, particularly among adult males in agricultural communities. In such cases of unknown causality, the condition has been dubbed 'CKD of uncertain aetiology'. Other emerging risk factors include pollution. ${ }^{34}$

Second, we observed substantial variability in the study inclusion criteria including age (range: 20.2-86 years) and sex (range: proportion of women included; $0 \%-77 \%$ ) as well as data collection methods and definitions used to define CKD (and formulas used to estimate GFR: Modification of Diet in Renal Disease Study, Chronic Kidney Disease Epidemiology Collaboration, Cockroft-Gault or others) across the region. In addition, a sizeable number of studies specifically included 'high-risk' patients (who tended to be older than those included in populationbased studies) in whom CKD would expect to be prevalent at higher frequency (eg, cardiovascular disease). The observed variability in CKD prevalence across Asia may thus be attributable to these differences in patient and study characteristics. Such results highlight the importance of consistent approaches to data collection regarding $\mathrm{CKD}$, and the value of longitudinal assessments to understand changes over time. In addition, the value of screening or early detection for CKD, and of interventions proven to prevent progression of CKD, as well as other complications, should be tested using novel models of care, with a particular focus on low-income and middle-income countries.

This study has several strengths. Our findings extend on previous estimates on CKD burden in the Asian region and to our knowledge, represents the first study to estimate the absolute number of people with CKD specifically in Asia using clustering analysis, an established statistical approach used to determine patterns of conditions. ${ }^{35} 36$ We obtained contemporary data from 16 countries, which encompasses $94.8 \%$ of the regional adult population, using a comprehensive and systematic approach. Some limitations should also be considered, mainly due to limited data availability, variability in the datasets used and their quality and reliability. In particular, there was substantial variation in individual study inclusion criteria, including studies 'high-risk' patients which may have contributed to an overestimation of CKD prevalence. While we sought to account for this variability by generating separate prevalence estimates using population-based studies only, there may be other potential sources of heterogeneity we have not considered.

We were unable to obtain CKD prevalence data for 10 countries (and had to rely on our estimates to obtain regional figures) while 41 studies (18.9\%) were assessed as having high risk of bias. However, given that these data limitations only pertained to a small proportion of the studied population (10 countries encompassing 5.2\% of the regional population; and that the 41 studies with high risk of bias comprised only $5.3 \%$ of the studied population in this review), the impact on the overall findings is likely small. In addition, the two countries with the largest populations in the region, China and India, do not have comprehensive data on national studies or CKD registries. As such, the potential for within-country variability arising from the limited availability of nationally representative data, cannot be excluded. As we used advanced CKD prevalence values in place of overall CKD prevalence where such estimates were unavailable, the total number of patients with CKD in these regions may have been underestimated. It is also possible that CKD prevalence is under reported as the number of publications we identified for some countries were relatively small. Most studies used only a single creatinine measurement to calculate eGFR, with no follow-up measurements to confirm CKD. This may have resulted in overestimation of CKD prevalence due to high false positive rates. Furthermore, while the clustering model we used to derive CKD prevalence in countries where no such estimates were available accounted for major risk factors associated with CKD such as diabetes and hypertension, there are likely additional factors (eg, other cardiovascular risk measures) which we were unable to account for due to limited availability of information.

CKD is a major, growing, health concern in Asia, particularly in China and India, affecting a substantial proportion of its population. Our study results highlight the need for a collaborative action in the region to combat CKD and its complications including CVD and death.

\section{Author affiliations}

${ }^{1}$ The George Institute for Global Health, Newtown, New South Wales, Australia ${ }^{2}$ School of Medicine, University of Sydney, Sydney, NSW, Australia

${ }^{3}$ Armadale Kelmscott Memorial Hospital, East Metropolitan Health Service, Perth, WA, Australia

${ }^{4}$ Department of Nephrology and Laboratory Medicine, Kanazawa University, Kanazawa, Japan

${ }^{5}$ The George Institute for Global Health, Imperial College London, London, UK ${ }^{6}$ Department of Epidemiology and Public Health, Graduate School of Medical Sciences, Kyushu University, Kyushu, Japan

${ }^{7}$ Faculty of Medicine and Health, University of New South Wales, Sydney, New South Wales, Australia

${ }^{8}$ Department of Epidemiology, Johns Hopkins Bloomberg School of Public Health, Baltimore, Maryland, USA 
${ }^{9}$ Division of Nephrology, Endocrinology, and Metabolism, Tokai University School of Medicine, Japan, Japan

${ }^{10}$ Division of Nephrology, Faculty of Medicine, Chulalongkorn University, Bangkok, Thailand

${ }^{11}$ Hospital Sultanah Aminah, Johor Bahru, Malaysia

${ }^{12}$ Okinawa Heart and Renal Association (OHRA) and Clinical Research Support

Center, Tomishiro Central Hospital, Okinawa, Japan

${ }^{13}$ Division of Nephrology, Department of Internal Medicine, Kaohsiung Medical University Hospital, Kaohsiung, Taiwan

${ }^{14}$ GlaxoSmithKline, Value Evidence Outcomes - Epidemiology, Stevenage,

Hertfordshire, UK

${ }^{15}$ The George Institute for Global Health, India, New Dehli, India

${ }^{16}$ Prasanna School of Public Health, Manipal Academy of Higher Education,

Manipal, India

${ }^{17}$ School of Public Health, Imperial College London, London, UK

Twitter Carinna Hockham @carinnahockham, Lai Seong Hooi @laihooi1 and Vivekanand Jha @vjha126

Acknowledgements TL was supported by an Australian Postgraduate Award. TT is supported by the Japan Society for the Promotion of Science Programme for Fostering Globally Talented Researchers. MJ was supported by a Scientia Fellowship from UNSW Sydney, Australia.

Contributors TL, TT, CH, TN and MJ were responsible for study concept design, data collection, analysis, interpretation and manuscript preparation. VJ, HS-F and VP were responsible for study concept design, interpretation and manuscript preparation, and all authors were involved in critical revision of the analyses, interpretation of the findings and the editing of manuscripts. The corresponding author (MJ) had full access to all data in the study and takes responsibility for the integrity of the data and accuracy of the analysis and final manuscript. This study was conducted as per methods previously described for ARC. The Steering Committee members for ARC include: VJ (India-Chair), VP (AustraliaDeputy Chair), Atsushi Wada (Japan), Daiki Inaguma (Japan), Heide StirnadelFarrant (UK), Helen Monaghan (Australia), Ho Jun Chin (South Korea), Hong Zhang (China), Hooi Lai Seong (Malaysia), Ikuto Masakane (Japan), Kamal Shah (India), Kazuhiko Tsuruya (Japan), Kearkiat Praditpornsilpa (Thailand), Kunihiro Matsushita (USA), Kunitoshi Iseki (Japan), Luxia Zhang (China), Mariko Miyazaki (Japan), Mark Woodward (Australia), Masafumi Fukagawa (Japan), Ming-Yen Lin (Taiwan), Rajasekara Chakravarthi (India), Satoshi Ogata (Japan), ShangJyh Hwang (Taiwan), Takayuki Hamano (Japan), Thaminda Liyanage (Australia), Toshiharu Ninomiya (Japan), Yoshinari Yasuda (Japan), Young-Hwan Hwang (Korea), Zuo Li (China).

Funding This study was funded by a research grant from GlaxoSmithKline (GSK) Limited. TL, TT, TN, VP, VJ, and MJ had access to all data. MJ and VJ were responsible for the decision to submit the manuscript.

Competing interests $\mathrm{TL}, \mathrm{TT}$ and $\mathrm{KP}$ have no relevant conflicts of interests to declare. MW declares consulting work for Amgen, Kyowa Kirin and Freeline, and was funded by an Australian National Health and Medical Research Council Investigator and Programme Grants. VP reports receiving personal fees for Advisory Boards or Scientific Presentations from Retrophin, Janssen, Merck, and Servier. VP also reports receiving fees for Advisory Boards, Steering Committee roles, or Scientific Presentations from Abbvie, Astellas, Astra Zeneca, Bayer, Baxter, BMS, Boehringer Ingelheim, Dimerix, Durect, Eli Lilly, Gilead, GSK, Janssen, Merck, Mitsubishi Tanabe, Novartis, Novo Nordisk, Pfizer, Pharmalink, Relypsa, Retrophin, Sanofi, Servier, Vifor and Tricida. HS-F was a full-time employee and shareholder of GlaxoSmithKline. VJ reports grant funding from NephroPlus, Baxter Healthcare and GSK and speaker fees and honoraria from Baxter Healthcare and AstraZeneca (all monies paid to the employing organisation). MJ reports receiving research support from (1) the Australian National Health and Medical Research Council and (2) VentureWise (a wholly-owned subsidiary of NPS MedicineWise) to conduct a project funded by AstraZeneca.

Patient consent for publication Not applicable.

Ethics approval This study was approved by the Human Research Ethics Committee of the University of Sydney (2015/217).

Provenance and peer review Not commissioned; externally peer reviewed.

Data availability statement All data relevant to the study are included in the article or uploaded as online supplemental information. All data generated or analysed during this study are included in this article and its online supplemental information files. Restrictions apply to the availability of individual participant data collected and used by agreement of the Asian Renal Collaboration for the current study, and so are not publicly available.

Supplemental material This content has been supplied by the author(s). It has not been vetted by BMJ Publishing Group Limited (BMJ) and may not have been peer-reviewed. Any opinions or recommendations discussed are solely those of the author(s) and are not endorsed by BMJ. BMJ disclaims all liability and responsibility arising from any reliance placed on the content. Where the content includes any translated material, BMJ does not warrant the accuracy and reliability of the translations (including but not limited to local regulations, clinical guidelines, terminology, drug names and drug dosages), and is not responsible for any error and/or omissions arising from translation and adaptation or otherwise.

Open access This is an open access article distributed in accordance with the Creative Commons Attribution Non Commercial (CC BY-NC 4.0) license, which permits others to distribute, remix, adapt, build upon this work non-commercially, and license their derivative works on different terms, provided the original work is properly cited, appropriate credit is given, any changes made indicated, and the use is non-commercial. See: http://creativecommons.org/licenses/by-nc/4.0/.

\section{ORCID iD}

Ming-Yen Lin http://orcid.org/0000-0002-8194-4833

\section{REFERENCES}

1 Inker LA, Astor BC, Fox CH, et al. KDOQI us commentary on the $2012 \mathrm{KDIGO}$ clinical practice guideline for the evaluation and management of CKD. Am J Kidney Dis 2014;63:713-35.

2 United States Renal Data System - 2012 Atlas of CKD \& ESRD 2012

3 Lozano R, Naghavi M, Foreman K, et al. Global and regional mortality from 235 causes of death for 20 age groups in 1990 and 2010: a systematic analysis for the global burden of disease study 2010. Lancet 2012;380:2095-128.

4 Jha V, Garcia-Garcia G, Iseki K, et al. Chronic kidney disease: global dimension and perspectives. Lancet 2013;382:260-72.

5 Liyanage T, Ninomiya T, Jha V, et al. Worldwide access to treatment for end-stage kidney disease: a systematic review. Lancet 2015;385:1975-82

6 Guariguata L, Whiting DR, Hambleton I, et al. Global estimates of diabetes prevalence for 2013 and projections for 2035. Diabetes Res Clin Pract 2014;103:137-49.

7 Nanditha A, Ma RCW, Ramachandran A, et al. Diabetes in Asia and the Pacific: implications for the global epidemic. Diabetes Care 2016;39:472-85.

8 Ranasinghe AV, Kumara GWGP, Karunarathna RH, et al. The incidence, prevalence and trends of chronic kidney disease and chronic kidney disease of uncertain aetiology (CKDu) in the North central province of Sri Lanka: an analysis of 30,566 patients. BMC Nephrol 2019;20:338.

9 Tatapudi RR, Rentala S, Gullipalli P, et al. High prevalence of CKD of unknown etiology in Uddanam, India. Kidney Int Rep 2019;4:380-9.

10 United Nations Economic and Social Commission for Asia and the Pacific. Ageing in Asia and the Pacific: overview, 2017. Available: https://www.unescap.org/resources/ageing-asia-and-pacificoverview

11 Liu FX, Rutherford P, Smoyer-Tomic K, et al. A global overview of renal registries: a systematic review. BMC Nephrol 2015;16:31.

12 Zhang L, Wang F, Wang L, et al. Prevalence of chronic kidney disease in China: a cross-sectional survey. Lancet 2012;379:815-22.

13 Imai E, Horio M, Watanabe T, et al. Prevalence of chronic kidney disease in the Japanese general population. Clin Exp Nephrol 2009;13:621-30.

14 Ito J, Dung DTK, Vuong MT, et al. Impact and perspective on chronic kidney disease in an Asian developing country: a large-scale survey in North Vietnam. Nephron Clin Pract 2008;109:c25-32.

15 Liyanage T, Ninomiya T, Perkovic V, et al. Chronic kidney disease in Asia: protocol for a collaborative overview. Nephrology 2017;22:456-62

16 United Nations Department of Economic and Social Affairs. Population division, population estimates and projections section. world population prospects: the 2019 revision. Available: http://esa. un.org/wpp

17 Stroup DF, Berlin JA, Morton SC, et al. Meta-Analysis of observational studies in epidemiology: a proposal for reporting. meta-analysis of observational studies in epidemiology (moose) group. JAMA 2000;283:2008-12.

18 KDIGO. Clinical practice guidelines for the evaluation and management of chronic kidney disease. Kidney Int Suppl 2012;2013:5-14. 
19 Levey AS, Bosch JP, Lewis JB, et al. A more accurate method to estimate glomerular filtration rate from serum creatinine: a new prediction equation. modification of diet in renal disease Study Group. Ann Intern Med 1999:130:461-70.

20 Levey AS, Stevens LA, Schmid CH, et al. A new equation to estimate glomerular filtration rate. Ann Intern Med 2009;150:604-12.

21 The global health Observatory data Repository website. Available: http://www.who.int/gho/ncd/risk_factors/en/

22 Bulletin of interior statistics, department of statistics, Ministry of the interior, Republic of China (Taiwan). Available: https://www.moi.gov. tw/files/site_stuff/321/2/year/year_en.html

23 Taiwan Ministry of Health and Welfare. Cause of death statistics. Available: https://www.mohw.gov.tw/np-128-2.html

24 Wells G, Shea B, O'Connell D. The Newcastle-Ottawa scale (NOS) for assessing the quality of nonrandomized studies in metaanalyses, 2009. Available: http://www.ohri.ca/programs/clinical_ epidemiology/oxford.htm

25 Dilts D, Khamalah J, Plotkin A. Using cluster analysis for medical resource decision making. Med Decis Making 1995;15:333-46.

26 McLachlan GJ. Cluster analysis and related techniques in medical research. Stat Methods Med Res 1992;1:27-48.

27 Fukuoka Y, Lindgren TG, Rankin SH, et al. Cluster analysis: a useful technique to identify elderly cardiac patients at risk for poor quality of life. Qual Life Res 2007;16:1655-63.
28 DeVon HA, Vuckovic K, Ryan CJ, et al. Systematic review of symptom clusters in cardiovascular disease. Eur J Cardiovasc Nurs 2017;16:6-17.

29 Kweon S, Kim Y, Jang M-jin, et al. Data resource profile: the Korea National health and nutrition examination survey (KNHANES). Int $J$ Epidemiol 2014;43:69-77.

30 Individual participant data provided by Min-Yen Lin (cross-sectional, general populated-based data from Taiwan).

31 Cockwell P, Fisher L-A. The global burden of chronic kidney disease. Lancet 2020;395:662-4.

32 Hasan M, Sutradhar I, Gupta RD, et al. Prevalence of chronic kidney disease in South Asia: a systematic review. BMC Nephrol 2018;19:291.

33 Zhang L, Long J, Jiang W, et al. Trends in chronic kidney disease in China. N Engl J Med 2016;375:905-6.

34 Li G, Huang J, Wang J, et al. Long-Term Exposure to Ambient PM and Increased Risk of CKD Prevalence in China. J Am Soc Nephrol 2021;32:448-58.

35 Cornell JE, Pugh JA, Williams, Jr JW, et al. Multimorbidity clusters: clustering binary data from multimorbidity clusters: clustering binary data from a large administrative medical database. AMR;12:163-82.

36 Everitt BS, Landau S, Leese M. Cluster analysis. 4th ed. NY: Oxford University Press, 2001. 